\title{
Delayed Onset of Muscle Soreness and The Activation of The Immune System
}

\author{
Mohammad Arif Ali ${ }^{1}$, Setya Rahayu ${ }^{2}$, Yang, Chia-En ${ }^{3}$, Nandaru Fajar Sumirat ${ }^{4}$, Bayu \\ Pangestu ${ }^{5}$, Gustiana Mega Anggita ${ }^{6}$, Sugiarto $^{7}$, Fuadah Nor Wiqoyatul Milla ${ }^{8}$ \\ \{hiarifalikhan@mail.unnes.ac.id ${ }^{1}$, setyarahayu@mail.unnes.ac.id ${ }^{2}$, yce@ mail.npust.edu.tw ${ }^{3}$ \} \\ Universitas Negeri Semarang, Semarang, Indonesia ${ }^{1,2}$ \\ National Pingtung University of Science and Technology, Pingtung, Taiwan ${ }^{3}$
}

\begin{abstract}
Inappropriate exercise prescription is one of many factors to sport injuries. Delayed onset of muscle soreness triggers the inflammatory processes. Therefore knowing the process of DOMS, physical exercise benefits, and the process of immune system activation after physical exercise will give us better understanding to create precise prescription. Original studies within last ten years were considered, other references were used only to support the storylines. The codifying/compilation stage, followed by analysis and synthesis, then analysis to synthesis were performed sequentially to provide acceptable answers. Eccentric contraction and high intensity exercise cause DOMS. DOMS sequences: mechanical damage; inflammation; and free radical proliferation. Physical exercise benefits both in physical and mental health. Neutrophil, macrophages as natural killer cells are types of the main cellular vulnerable congenital of immune system, changed because of physical exercise. Proper methods and intensity in physical exercise are keys to activates immune system.
\end{abstract}

Keywords: physical exercise; DOMS; immune system.

\section{Introduction}

The human immune system has important and complex task in human survival. The immune system can identify harmful microbes and microbes that are beneficial to the human body [1]. Physical exercise has become a necessity for every individual in this modern era, it is because to increase the immune system in the human body is one of physical exercise purposes. Once immune system become stronger, it leads to improve health [2].

Unfortunately, besides having positive effects, acute physical exercise could brings undesirable effects (injury) especially when physical exercise is not following the proper exercise presription. Athletes are including in high risk of injury, when prolonged physical exercise or too heavy exercise is performed [3]. Injury causes muscle trauma, based on the type of trauma or muscle strain are classified into three grades: grade I (Mild damage to individual muscle fibers), grade II (More extensive damage with more muscle fibers involved), type III (Complete rupture of a muscle or tendon). Grade I strain is characterized by microscopic damage to muscle fibers 
which then results in pain and swelling [4]. Muscles will respond to increased intensity of exercise with pain, and this type of pain is commonly considered as Delayed Onset of Muscle Soreness or DOMS [5].

Based on the description above, the objectives of this study are: 1) To describe the process of delayed onset of muscle soreness as part of physical exercise effects. 2) To elaborate on the benefits of physical exercise. 3) To describe the process of immune system activation after physical exercise.

\section{Materials and Methods}

Materials, this is a narrative review, data in this paper are secondary. Mainly original studies within last ten years of being published were considered on this paper, additionally other references were being used only to support the storylines in order to answer the research objectives. Materials were obtained from data stations such as Google Scholar, PubMed, ResearchGate, Reports, Blogspots, News Paper, etc. Twenty two papers cited in this study, eighty papers $(82 \%)$ were published between years 2010 to 2020; two papers (9\%) year 2009 and 2003; last two papers (9\%) year 1993 and 1991.

Methods, there are three stages must be done sequentially in order to provide acceptable answers for the questions. 1) The Codifying or Compilation Stage is when investigators doing collection and sorting all related materials from sources. 2) Analysis and Synthesis Stage, profound and detailed examination (extracting data) on how data from chosen materials could be constructed into data interpretation. 3) Conclusion Drawing is the last stage to end the process of literature study and to state the novelty of study.

\section{Results and Discussion}

Since this is a narrative review which all data in this paper are secondary, the limitations we do have is more than systematic review study. In this paper we only focus on specific issue, there are DOMS, physical exercise benefits, and the activation of immune system post exercise.

\subsection{DOMS as acute effect of physical exercise}

DOMS is ultrastructural muscle damage, it is often associated with increased pain, stiffness, swelling, and mechanical changes in the joints [7]. DOMS causes pain in the muscles and will worsen when used to contract [8]. DOMS occurs because of extra muscle contraction and also a contration which is not usually done by the people called eccentric contraction, DOMS is also considered as strain grade I [9].

Muscle stiffness due to DOMS can limit movement (decreased range of motion) and interfere onto daily activities (Pearcey et al., 2015). DOMS not only attacks an athlete, but DOMS also might be experiened by untrained people [10] or people who engage in eccentric physical exercise [7]. DOMS occurs within 12 hours and then 
peaks within 24 to 72 hours after practice and takes five to seven days to heal [10], [11].

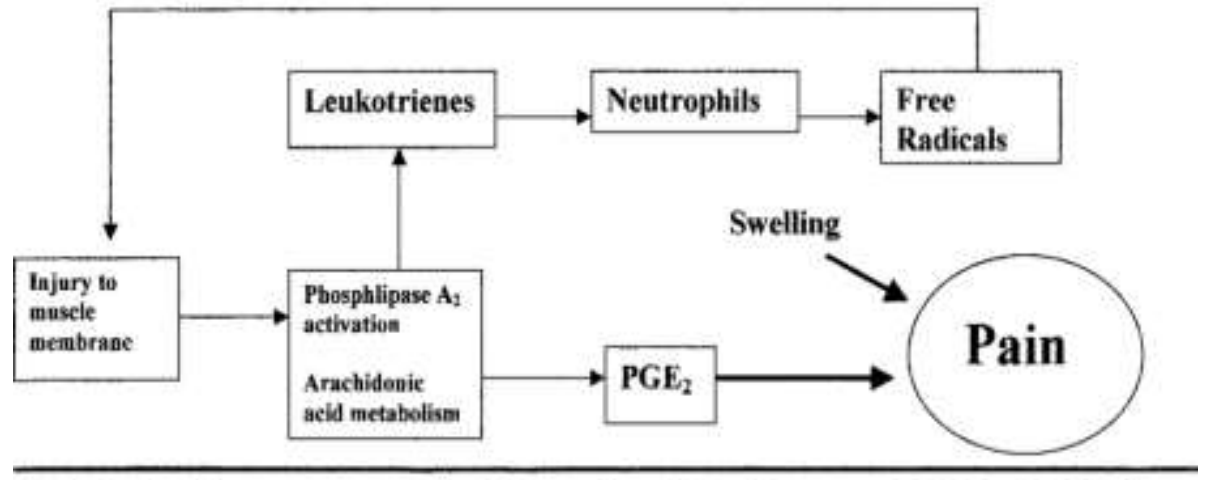

I. Mechanical Damage

II. Inflammation \& Swelling

III. Free Radical

Proliferation

Fig. 1. Schematic showing possible sequence of injury of delayed onset muscle soreness. Figure is adopted from Connolly, Declan A.J., Sayers, Stephen E., Mchugh, Malachy P., 2003 [6].

Inflammation processes occur underlying DOMS condition, this process involving leukocytes that is remarkable biomarker of DOMS [12]. Leukocytes consisting of neutrophils, lymphocytes, basophil, eosinophils, and monocytes [13]. Monocytes are one of the most important in the process of inflammatory [14]. Microscopic damage on muscle fibers occurred because DOMS, and then there is the repair process conducted by muscle fibers monocytes after receiving signals from microscopic damage to work on the process of angiogenesis and fibrosis [15]. Mechanical damage is common initiation cause DOMS (there is an injury from performing physical exercise or sport to muscle membrane), then followed by inflammation (swelling in physical apperance) activation as body response, the end what cause the pain sensation is free radical proliferation, (Figure 1).

\subsection{Benefits of physical exercise on health}

Physical exercise is useful to maintain muscle strength especially in elderly and reduced risk of death caused by diseases [17]. Moderate exercise can reduces the risk of chronic diseases in human, including cardiovascular disease, type 2 diabetes, and cancer [18]. Physical exercise affecting our immune system, particularly moderate exercise can provides positive effects on immune system improvement [19]. Furthermore, physical exercise also has many benefits on mental health management, start from improving mental health, improving mood, and neuroplasticity using the factor neutropic of the brain [20]. Less of physical activity increased risk of health problems [18]. 


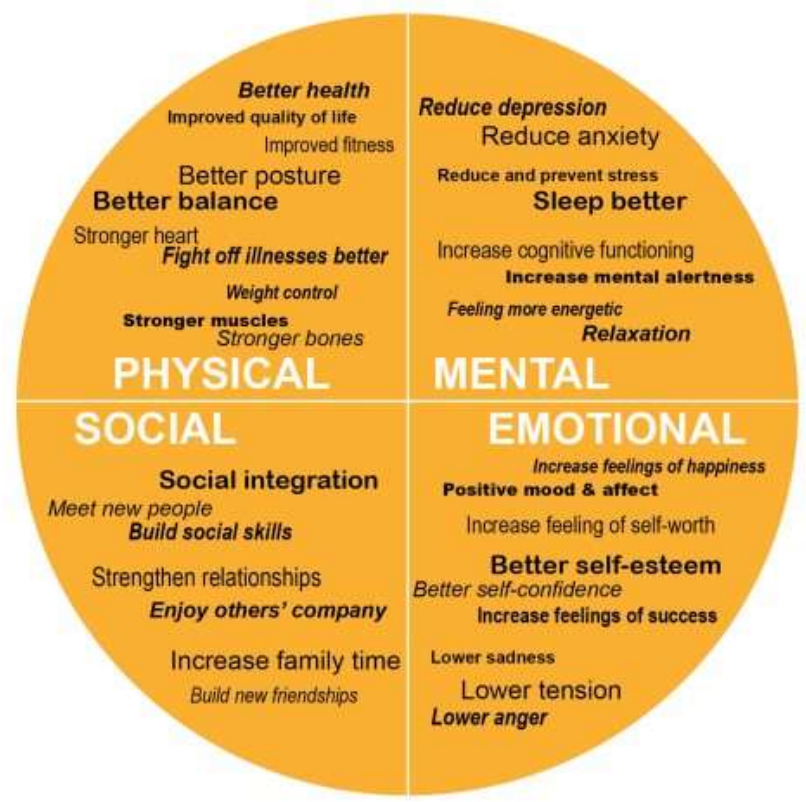

Fig. 2. Benefits of physical exercise on physical, mental, social, and emotional health. Figure is adopted from https://www.enoughwiththemadness.com/blog/the-benefits-of-physical-activityand-so-much-more [16].

\subsection{Sports activates immune}

The effect of exercise on immune system varies different depending on the intensity and methods used for physical exercise. The higher intensity would give better results for good immune when it is conducted with the right prescription. However, if it is mistaken, then the risk of injury become higher. For example, is boxing and running, they have different risk of injury. Sports can influence the immune system in a different way [21].

Regular physical activity can be diversifying the act of anti-inflammatory. Cooperation between organs that are mediated by physical activity set the leading in improving cytokines anti-inflammatory and declines in a pro-inflammatory cytokine. When muscle contract, myokin takes part in sent down chronic inflammation. Particles of a muscle that was revealed by interleukin recognized as myokin apleiotropic in modulation metabolism immune and inflammatory [20]. The immune main defense system is body against pathogenic. Neutrophil, macrophages a natural killers are the typea of the main cellular vulnerable congenital of immune system, changes because of physical exercise. Then neutrophils capable of killing bacteria through the release of enzymes and through the secretion of reactive oxygen species .

According to Pedersen's investigation about the effect of static cycling on ergometer cycle for 60 minutes in a trained and healthy young man, with target intensity $75 \%$ of VO2Max. Showed that acute effect of physical exercise is very influential to 
improve human immunity (increased leukocytes, lymphocytes, and neutrophil). This is one of the evidence showing that exercise is actually a challenge to our body, (Table 1).

Table 1. Effects of ergometer cycling for 60 minutes (75\% VO2 MAX). Table is adopted from Pedersen, 1991.

\begin{tabular}{lll}
\hline Immune Agents & During Exercise & 2h Post Exercise \\
\hline Leukocytes $\left(10^{3} / \mu 1\right)$ & $\uparrow$ Increased & $\uparrow$ Increased \\
Lymphocytes $(\%)$ & $\uparrow$ Increased & $\uparrow$ Increased \\
Neutrophil $(\%)$ & $\uparrow$ Increased & $\uparrow$ Increased \\
\hline
\end{tabular}

Leukocytes are human defense system or commonly called white blood cells. Leukocytes consist of neutrophils, lymphocytes, basophils, eosinophils, and monocytes [13]. The increase of leukocytes is needed by the body as the immune system. Moreover, a study conducted by Laeto showing the effect of physical exercise on changes in the number of leukocytes. Subjects in the study were trained young men with an age range of 18-24 years old. Physical exercise performed for 60 minutes can increase the number of leukocytes. This increase is caused by stress and the burden received by the body due to physical exercise. This also relates to blood transfer activities from lymph vessels to blood vessels. Data are shown in table 2 below:

Table 2. Effects of physical exercise on leucocyte levels in trained young men. Table is adopted from Laeto et al., 2017.

\begin{tabular}{lllc}
\hline Immune Agents & $\begin{array}{l}\text { Average } \pm \\
\text { before the intervention }\end{array}$ & $\begin{array}{l}\text { Average } \pm \\
\text { after the intervention }\end{array}$ & $\boldsymbol{P}$ \\
\hline Leukocytes $\left(10^{3} / \mu \mathrm{l}\right)$ & $7912 \pm 968$ & $9387 \pm 2736$ & 0,013 \\
Neutrophils $(\%)$ & $4359 \pm 718$ & $6462 \pm 2783$ & 0,005 \\
Eosinophils (\%) & $206 \pm 130$ & $162 \pm 122$ & 0,024 \\
Basophils $(\%)$ & $53 \pm 20$ & $55 \pm 16$ & 0,493 \\
Lymphocytes $(\%)$ & $2689 \pm 480$ & $2107 \pm 649$ & 0,002 \\
Monocytes $(\%)$ & $605 \pm 124$ & $601 \pm 235$ & 0,928 \\
\hline
\end{tabular}

Additionally, a reduction of normal functioning immune because of age is called Immunosenescence. It is marked by impaired immune function cellular and an increase in inflammatory activity. Age affects the innate immune system (macrophages, neutrophil, the dendrites, and natural killer cells) and adaptive immune system (lymphocytes-T and lymphocyte-B). However, Increasing physical exercise can increase sensitivity to infection (body is ready to combat any pathogen) [17].

\section{Conclusion}

In brief, sequences of DOMS are 1) Mechanical damage, 2) ilflammation/swelling, 3) Free radical proliferation. Physical exercise benefits both in physical health and mental health. The proper intensity and methods used for physical exercise 
is a key to activates our immune system. Neutrophil, macrophages as natural killer cells are the types of the main cellular vulnerable congenital of immune system (the innate immune system), changes because of physical exercise.

Acknowledgments. This study is part of an investigation about effects of high density foam roller on leucocytes and monocytes levels as indicators of delayed-onset muscle soreness (DOMS) post long distance running in trained young males. This study was granted by Faculty of Sports Science, Universitas Negeri Semarang (DIPA FIK UNNES Tahun Anggaran 2020. Nomor Surat Perjanjian Penugasan: 2.4.5/UN37/PPK.3.1/2020).

\section{References}

[1]. S. K. Lundy, "The immune system, in sickness \& in health - part 1: microbes and vaccines,” Immune Syst. Fight. Infect. Microbes, vol. 6, no. September, pp. 1-7, 2018.

[2]. P. Krustrup, J. Dvorak, and J. Bangsbo, "Small-sided football in schools and leisure-time sport clubs improves physical fi tness, health pro fi le, well-being and learning in children,” Br. J. Sports Med., vol. 50, no. 19, pp. 1166-1167, 2016.

[3]. A. W. Ilmawan, "The effect of doms massage on pain reduction and increased range of motion (ROM) and function in the case of delayed onset muscle soreness (DOMS) in the legs," Universitas Negeri Yogyakarta, 2018.

[4]. D. Delos, T. G. Maak, and S. A. Rodeo, "Muscle injuries in athletes: enhancing recovery through scientific understanding and novel therapies," Sport Heal., vol. 5, no. 4, pp. 346352,2013 .

[5]. [5] P. B. Lewis and D. Ruby, "Muscle soreness and delayed-onset muscle," CSM, vol. 31, no. 2, pp. 255-262, 2012.

[6]. M. M. H. D. Connolly, S. Ayers, "Treatment and prevention of delayed onset," J. Strength Cond. Res., vol. 17, no. 1, pp. 197-208, 2003.

[7]. T. Hotfiel et al., "Advances in delayed-onset muscle soreness (DOMS): part i: pathogenesis and diagnostics delayed onset muscle soreness - teil i: pathogenese und diagnostik authors mechanisms and pathogenesis," Sport. Sport., vol. 32, no. 4, pp. 243250, 2018.

[8]. L. Stefanelli et al., "Delayed-onset muscle soreness and topical analgesic alter corticospinal excitability of the biceps brachii," Med. Sci. Sport. Exerc., vol. 51, no. 11, pp. 2344-2356, 2019.

[9]. R. Heiss, C. Lutter, J. Freiwald, M. W. Hoppe, C. Grim, and K. Poettgen, "Advances in delayed-onset muscle soreness ( DOMS ) - part ii : treatment and prevention delayed onset muscle soreness - teil ii : therapie und prävention authors," Sport. Sport., vol. 33, no. 01, pp. 21-29, 2019.

[10]. G. E. P. Pearcey, D. J. Bradbury-squires, E. J. Drinkwater, D. G. Behm, and C. Duane, "Foam rolling for delayed-onset muscle soreness and recovery of dynamic performance measures," J. Athl. Train., vol. 50, no. 1, pp. 5-13, 2015.

[11]. S. P. Selkar, G. J. Ramteke, and A. K. Dongare, "Effect of eccentric muscle training to reduce severity of delayed onset muscle soreness in athletic subjects," Eur. J. Gen. Med., vol. 6, no. 4, pp. 213-217, 2009. 
[12]. I. G. Fatouros and A. Z. Jamurtas, "Insights into the molecular etiology of exerciseinduced inflammation: opportunities for optimizing performance," J. Inflamation Res., vol. 9, pp. 175-186, 2016.

[13]. B. K. Pedersen, "Influence of physical activity on the cellular immune system: mechanisms of action," J. Sport Med., vol. 12, no. 18, pp. 23-29, 1991.

[14]. A. R. Jajtner et al., "Mediators of monocyte migration in response to recovery modalities following resistance exercise," J. Mediat. Inflamm., vol. 2014, pp. 1-9, 2014.

[15]. T. M. Best, B. Gharaibeh, and J. Huard, "Stem cells, angiogenesis and muscle healing : a potential role in massage therapies," Postgrad. Med. J., vol. 89, no. 1057, pp. 666-670, 2013.

[16]. "The benefits of physical activity and so much more," 2019. [Online]. Available: https://www.enoughwiththemadness.com/blog/the-benefits-of-physical-activity-and-somuch-more.

[17]. V. Valdiglesias et al., "Immune biomarkers in older adults : role of physical activity immune biomarkers in older adults : role of physical activity," J. Toxicol. Environ. Heal. Part A, vol. 00, no. 00, pp. 1-16, 2017.

[18]. S. Estruel-amades, M. Camps-bossacoma, M. Malén, J. P. Francisco, and M. Castell, "Alterations in the innate immune system due to exhausting exercise in intensively trained rats," pp. 1-12, 2020.

[19]. G. P. Tilz et al., "Increased immune activation during and after physical exercise," Immunibiology, vol. 188, no. 1-2, pp. 194-202, 1993.

[20]. R. Codella, L. Luzi, and I. Terruzzi, "Exercise has the guts: How physical activity may positively modulate gut microbiota in chronic and immune-based diseases," Dig. Liver Dis., vol. 50, no. 4, pp. 331-341, 2018.

[21]. J. G. B. Van Dijk and K. D. Matson, "Integrative and comparative biology ecological immunology through the lens of exercise immunology: new perspective on the links between physical activity and immune function and disease susceptibility in wild animals," vol. 56, no. 2, pp. 290-303, 2016.

[22]. A. Bin Laeto, R. Natsir, and M. A. Arsyad, "Effect of night futsal on the number of leukocytes and the count of leukocytes in young adults," pp. 35-40, 2017. 